\title{
Synthesis of Defect-Fluorite Pyrochlore Sodium Niobate Nanoparticles and Characterization of their Tolerance to Neutron Radiation
}

\author{
Rana Faryad Ali ${ }^{1}$, Jeffrey S. Ovens ${ }^{1}$, Krzysztof Starosta ${ }^{1}$, Byron D. Gates ${ }^{1 *}$ \\ 1. Department of Chemistry and 4D LABS, Simon Fraser University, 8888 University Drive, Burnaby, \\ BC, V5A 1S6, Canada \\ * Corresponding author: bgates@ sfu.ca
}

Radiation-resistant materials are sought for use in applications that present hostile environments, such as within nuclear reactors, and immobilization or transport of nuclear waste. Radiation resistance is measured as the ability of the materials to tolerate undesirable radiation-induced phenomena such as amorphization, swelling, cavitation, formation of new crystalline phases, and the clustering of point defects [1]. The materials used in applications for which they must resist damage from radiation exposure must be thermally resilient, chemically durable, and mechanically tolerant to the effects of radiation damage [2]. The main source of radiation in a nuclear reactor are energetic neutrons produced by fission and fusion reactions of a sufficient kinetic energy (e.g., > $1 \mathrm{MeV}$ ), which can damage a material by depositing energy into its lattice by collisions with atoms therein. Fast neutrons (e.g., neutrons with energies $>1 \mathrm{MeV}$ ) passing through a material can displace its atoms, which can lead to structural damage in the material (e.g., vacancies, amorphization, and other defects) [3].

Pyrochlore based materials have been widely explored for their relatively high tolerance to radiation damage, their phase stability at elevated temperatures, and their ability to effectively retain nuclear waste. Materials possessing a disordered or defect-fluorite pyrochlore structure are promising candidates to resist the detrimental effects of radiation damage [4]. Defect-fluorite pyrochlore materials have a higher radiation tolerance, thermal stability, and chemical durability relative to that of ordered or defect-free pyrochlores. This radiation tolerance of the defect-fluorite pyrochlores is due to its ability to accommodate lattice disorder and to recover from point defects as a result of cation mixing in their crystal lattice [5].

In this report, we demonstrate for the first time the synthesis and a detailed analysis of defect-fluorite pyrochlore sodium niobate $\left(\mathrm{NaNbO}_{3}\right)$ nanoparticles. A surfactant-assisted solvothermal method is used to prepare nanoparticles of $\mathrm{NaNbO}_{3}$. The solution-phase process to prepare these nanoparticles was carried out at a relatively low temperature (e.g., $220^{\circ} \mathrm{C}$ ) over a reaction time of $96 \mathrm{~h}$. The reagents were dissolved in benzyl alcohol, and trimethylamine was added as a surfactant. Morphology, phase, purity, and composition of the product were characterized by transmission electron microscopy (TEM), X-ray diffraction (XRD), Raman spectroscopy, and energy dispersive X-ray spectroscopy (EDS) analyses. Rietveld refinements of the XRD data from the as-synthesized product indicated the formation of a pure, defect-fluorite pyrochlore of $\mathrm{NaNbO}_{3}$ having a cubic crystal structure associated with the $F m \overline{3} m$ space group. Variable temperature XRD indicated that these defect-fluorite pyrochlore nanoparticles were stable up to at least $500{ }^{\circ} \mathrm{C}$. At higher temperatures the nanoparticles transformed into the cubic and rhombohedral perovskite phases of $\mathrm{NaNbO}_{3}$ along with the introduction of a pseudo-hexagonal $\mathrm{Nb}_{2} \mathrm{O}_{5}$. The as-synthesized nanoparticles had an average diameter of $\sim 74 \pm 11 \mathrm{~nm}$ as observed by TEM analysis. These defect-fluorite pyrochlore nanoparticles of $\mathrm{NaNbO}_{3}$ also exhibited a resistance to radiation induced amorphization. Stability of the nanoparticles to fast neutrons was studied by irradiating the sample with 
14.1 MeV neutrons for $1 \mathrm{~h}$. The dimensions, phase, composition, and crystallinity of the defect-fluorite pyrochlore nanoparticles after exposure to this flux of neutrons were relatively comparable to the assynthesized product. The radiation tolerance of these pyrochlore nanoparticles could be useful in the design of radiation tolerant materials for the handling and storage of radioactive waste [6].

\section{References:}

[1] Grimes, et al., Nat. Mater. 7 (2008), p. 683.

[2] Sickafus, et al., Science 289 (2000), p. 748.

[3] Zinkle, et al., Mater. Today 12 (2009), p. 12.

[4] Sickafus, et al., Nat. Mater. 6 (2007), p. 217.

[5] Lumpkin, et al., Chem. Mater. 21 (2009), p. 2746.

[6] This research was financially supported in part by from the Natural Sciences and Engineering Research Council (NSERC) Discovery Program (Grant No. 1077758), CMC Microsystems (MNT Grant Program), and the Canada Research Chairs Program (B.D. Gates; Grant No. 950-215846). This work made use of 4D LABS (www.4dlabs.ca) and the Centre for Soft Materials shared facilities supported by the Canada Foundation for Innovation (CFI), British Columbia Knowledge Development Fund (BCKDF), Western Economic Diversification Canada, and Simon Fraser University.

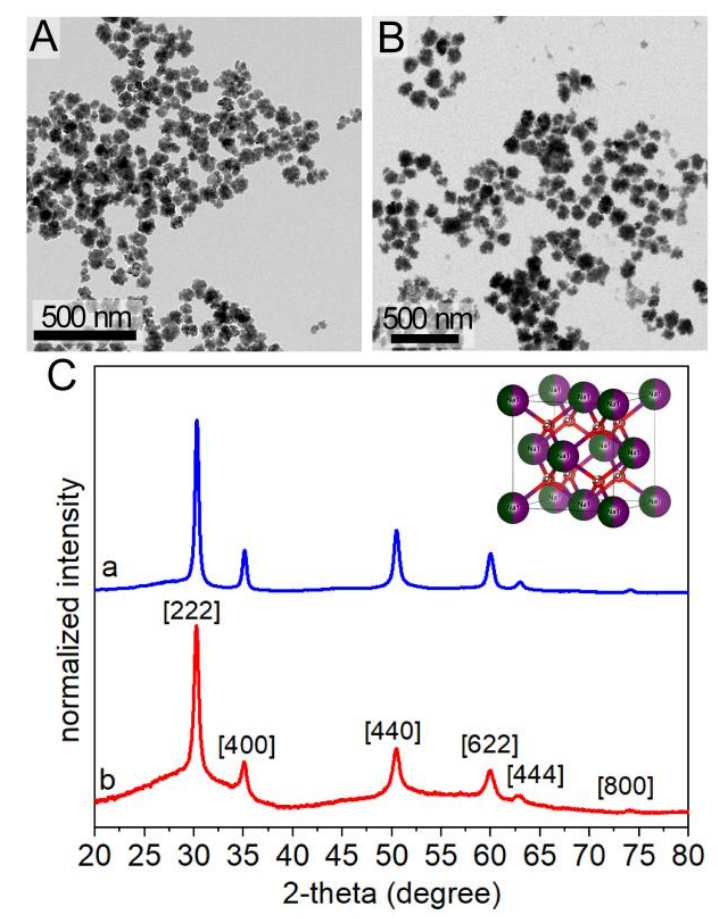

Figure 1. (A,B) Transmission electron microscopy (TEM) analyses of (A) the as-prepared defect-fluorite pyrochlore $\mathrm{NaNbO}_{3}$ nanoparticles before exposure to neutrons and (B) the $\mathrm{NaNbO}_{3}$ nanoparticles after neutron irradiation for $1 \mathrm{~h}$. (C) Powder X-ray diffraction patterns of the nanoparticles (a) before, and (b) after neutron irradiation. 